\title{
A Method of Solving the Eigenproblem of the Atomic Ion Hamiltonians. I. Theory
}

\author{
J. OWEDYK ${ }^{*}, \|$
}

Faculty of Technology, Kujawy and Pomorze University, Bydgoszcz, Poland

(Received September 30, 2012; in final form March 26, 2014)

\begin{abstract}
The aim of this paper is to present a procedure for determining the power series in $\frac{N-1}{Z}$ for the approximate energy levels and eigenspaces of the nonrelativistic Hamiltonians $H^{(N, Z)}$ with $N$-electrons and nuclear charge $Z$. To this effect the theory of the best multiconfigurational approximations is applied.
\end{abstract}

DOI: 10.12693/APhysPolA.125.1075

PACS:

\section{Introduction}

\subsection{General comments}

A useful approximation method (the best multiconfigurational approximations) for solving the eigenproblem in the lower part of the spectrum of the Hamiltonian of $N$ identical fermions was proposed by Pruski [1]. This method relies on (i) finding the so-called minimizing basic manifold $\mathcal{M}$, which is a finite-dimensional space of 1-fermion functions and minimizes the average energy of the $N$-fermion system in the $N$-fold Grassman product $\mathcal{M}^{\wedge N}$ of $\mathcal{M}\left(\mathcal{M}^{\wedge N}\right.$ is a linear hull of all Slater determinants of $N$ 1-fermion functions from $\mathcal{M}$ ) and (ii) constructing in $\mathcal{M}^{\wedge N}$ a set of three successive approximation Hamiltonians of the real system. These three successive Hamiltonians are: the best 1-particle approximation, the best coarse-grained configurational approximation and the full multiconfigurational picture of the system. Each of them greatly facilitates the construction of the next one. Moreover, each successive model is better than the previous one.

The purpose of the present paper is to use the best approximations for the atomic ions system. The characteristic feature of the atomic ions is that atomic ions $(N, Z)$ having the same reduced nuclear charge $\xi=\lambda^{-1}=\frac{Z}{N-1}$ have the same minimizing basic manifolds [2]. The minimizing basic manifold and eigenvalues of the best approximations are calculated by means of a method similar to the perturbation theory. Here we use as a perturbation parameter $\lambda=\frac{N-1}{Z}$ which is contained in the interval $\langle 0,1)$, for each atomic ion. The case $\lambda=0$ is fulfilled for one-electron ions in which eigenproblem is solved exactly. In this way, through the expansion of eigenfunctions and eigenvalues in powers of $\lambda$, we obtain perturbation expansions which are valid for each $\lambda \in\langle 0,1)$, i.e. for all atomic ions. The coefficients of these expansions are universal, independent of $N$ and $Z$ quantities, and depend on the dimension of the minimizing basic manifold only.

\footnotetext{
*e-mail: j.owedyk@kpsw.edu.pl

॥ Address for correspondence: Jan Owedyk, Koszalińska 23A, 85-714 Bydgoszcz, Poland
}

The calculations are carried out by means of the canonical transformations method, which enables us to present eigenspaces by their projectors [3]. These calculations are invariant under the three-dimensional rotation group (symmetry group of atomic ion Hamiltonians). As a consequence the spherical harmonics of wave functions are not used in calculations. Concerning the canonical transformation $U$ we postulate that $U=\exp (\mathrm{i} D)$ where $D$ is a self-adjoint operator which is expanded in a power series in $\lambda$, i.e. $D=D_{\lambda}=\lambda D_{1}+\lambda^{2} D_{2}+\ldots$ The operator $D_{\lambda}$ is uniquely determined if we additionally require that operators $D_{1}, D_{2}, \ldots$ have the Hilbert-Schmidt norm minimal. This condition is equivalent to a minimum distance between perturbed and unperturbed eigenfunctions. Moreover, operators $D_{1}, D_{2}, \ldots$ are invariant under the three-dimensional rotation group.

At the very end of this paper the final formulae for the approximation energies of atomic ions in the case of the best 1-particle approximation and the best coarse-grained configurational approximation are presented.

\subsection{Some definitions}

Let $\mathcal{H}$ be the Hilbert space of square-integrable 1-particle functions, $\mathcal{H}^{\wedge N}$ that of square-integrable $N$-particle functions associated with a system of $N$ fermions. For $N \geq 2, \mathcal{H}^{\wedge N}=\mathcal{H} \wedge \ldots \wedge \mathcal{H}(N$ times $)$ is conceived as the $N$-fold Grassman product of $\mathcal{H}$ by itself, i.e. the subspace $A(N) \mathcal{H}^{N}$ consisting of completely antisymmetric elements of the $N$-fold tensor product $\mathcal{H}^{N}=\mathcal{H} \otimes \ldots \otimes \mathcal{H}(N$ times $)$ of $\mathcal{H}$ by itself, where $A(N)$ is the antisymmetric projector. The Hilbert spaces $\mathcal{H}^{N}$ and $\mathcal{H}^{\wedge N}$ are spanned, respectively, by their simple elements $f_{1} \otimes f_{2} \otimes \ldots \otimes f_{N}$ and $f_{1} \wedge f_{2} \wedge \ldots \wedge f_{N}=A(N)$ $\left(f_{1} \otimes f_{2} \otimes \ldots \otimes f_{N}\right)$.

If $K(p)$ and $L(q)$ are two operators in $\mathcal{H}^{p}$ and $\mathcal{H}^{q}$, $K(p) \otimes L(q)$ is an operator in $\mathcal{H}^{p+q}$, and the projection of the latter onto $\mathcal{H}^{\wedge(p+q)}$ is denoted by $K(p) \wedge L(q)$, i.e. $K(p) \wedge L(q)=A(p+q)(K(p) \otimes L(q)) A(p+q)$. It is easily found that $K(p) \wedge L(q)=\hat{K}(p) \wedge \hat{L}(q)=L(q) \wedge$ $K(p)$, where $\hat{K}(p)=A(p) K(p) A(p)$ is an antisymmetric projection of the operator $K(p)$ onto $\mathcal{H}^{\wedge p}$. The symbol $K^{\wedge m}$ is defined as $K^{\wedge m}=K \wedge K \wedge \ldots \wedge K$ ( $m$ times $)$, where $K(l)$ is an operator in $\mathcal{H}^{\wedge l}$ and $K^{\wedge m}$ is an operator 
in $\mathcal{H}^{\wedge l m}$. In particular, if $I=I(1)$ is the identity in $\mathcal{H}$, the operator $I(N)=I \otimes I \otimes \ldots \otimes I$ ( $N$ times) is an identity in $\mathcal{H}^{N}$ and $\hat{I}(N)=A(N) I(N) A(N)=I^{\wedge N}=A(N)$ is an identity in $\mathcal{H}^{\wedge N}$.

It is often convenient to use the following indicial (or tensorial) notation [2]. This notation is connected with many-particle calculations. We denote $f^{1}, f^{2}, f^{3}, \ldots$ for the elements of $\mathcal{H}$, and $f^{12 \ldots N}, f^{1^{\prime} 2^{\prime} \ldots N^{\prime}}, \ldots$ for those of $\mathcal{H}^{N}$. The complex conjugates to $f^{1}$ and $f^{12 \ldots N}$ are denoted by $f_{1}=\overline{f^{1}}$ and $f_{12 \ldots N}=\overline{f^{12 \ldots N}}$. The indices in $f^{12 \ldots N}$ correspond to the particles $1,2, \ldots, N$. The Einsteinian convention of summation is adopted. In particular, the expression

$$
f^{12 \ldots N} g_{12 \ldots N}=\langle f \mid g\rangle
$$

is the scalar product of $f$ and $g$. If $\langle f \mid f\rangle=1$, the symbol

$$
P_{1^{\prime} 2^{\prime} \ldots N^{\prime}}^{12 \ldots N}=f^{12 \ldots N} f_{1^{\prime} 2^{\prime} \ldots N^{\prime}}
$$

represents the projector onto $f \in \mathcal{H}^{N}$. In fact, for any $g^{12 \ldots N}$ :

$$
\begin{aligned}
& P_{1^{\prime} 2^{\prime}, \ldots N^{\prime}}^{12 \ldots N} g^{1^{\prime} 2^{\prime} \ldots N^{\prime}}=f^{12 \ldots N} f_{1^{\prime} 2^{\prime} \ldots N^{\prime}} g^{1^{\prime} 2^{\prime} \ldots N^{\prime}} \\
& =\langle g \mid f\rangle f^{12 \ldots N},
\end{aligned}
$$

which is characteristic for the projector onto $f$. If $\{f\}_{i \in I}$ is an orthonormal set in $\mathcal{H}^{\wedge N}$ (i.e. $\left.f_{(i)}^{12 \ldots N} \underset{(k)}{f_{12 \ldots N}}=\delta_{i, k}\right)$, then

$$
P_{1^{\prime} 2^{\prime} \ldots N^{\prime}}^{12 \ldots N}=\sum_{i \in I} f_{(i)}^{12 \ldots N} f_{1^{\prime} 2^{\prime} \ldots N^{\prime}}
$$

defines the projector onto the subspace $\operatorname{lin}\{f\}_{i \in I} \subset$ (i) $\mathcal{H}^{\wedge N}$. In particular if $\{f\}_{i \in I}$ is complete in $\mathcal{H}^{\wedge N}$, then (i)

$P(N)=\hat{I}(N)$. If $\{\underset{(i)}{f}\}_{i \in I}$ is the orthonormal base in the subspace $\mathcal{M} \subset \mathcal{H}$, then

$$
P_{1^{\prime}}^{1}=\sum_{i \in I} f_{(i)}^{1} f_{1^{\prime}}
$$

is the projector onto $\mathcal{M}$ and

$$
(P \wedge P)_{1^{\prime} 2^{\prime}}^{12}=\frac{1}{2}\left(P_{1^{\prime}}^{1} P_{2^{\prime}}^{2}-P_{2^{\prime}}^{1} P_{1^{\prime}}^{2}\right)
$$

represents the projector onto $\mathcal{M}^{\wedge 2}=\mathcal{M} \wedge \mathcal{M} \subset \mathcal{H}^{\wedge 2}$ and in general

$$
\left(P^{\wedge N}\right)_{1^{\prime} 2^{\prime} \ldots N^{\prime}}^{12 \ldots N}=\frac{1}{N !}\left|\begin{array}{c}
P_{1^{\prime}}^{1} P_{2^{\prime}}^{1} \ldots P_{N^{\prime}}^{1} \\
P_{1^{\prime}}^{2} P_{2^{\prime}}^{2} \ldots P_{N^{\prime}}^{2} \\
\ldots \\
P_{1^{\prime}}^{N} P_{2^{\prime}}^{N} \ldots P_{N^{\prime}}^{N}
\end{array}\right|
$$

is the projector onto $\mathcal{M}^{\wedge N} \subset \mathcal{H}^{\wedge N}$. We assume that the linear operators to be dealt with are representable as integral operators. The kernels of the latter are generalized functions of particle coordinates and will be written in the form $K_{1^{\prime} 2^{\prime}}^{12 \ldots}{ }_{N^{\prime}} . K$ is linear operator in the Hilbert space $\mathcal{H}^{\wedge N}$ of $N$-fermions and takes the form

$$
g^{12 \ldots N}=K_{1^{\prime} 2^{\prime} \ldots N^{\prime}}^{12 \ldots f^{1}} f^{1^{\prime} 2^{\prime} \ldots N^{\prime}} .
$$

If the kernel $K_{1^{\prime} 2^{\prime} \ldots N^{\prime}}^{12 \ldots N^{\prime}}$ is self-adjoint, then, obviously $\overline{K_{1}^{\prime} 2^{\prime} \ldots N^{\prime}}=K_{12 \ldots N}^{1^{\prime} 2^{\prime} \ldots N^{\prime}}$ and Eq. (1.2.8) may be written equivalently in the form

$$
g_{12 \ldots N}=K_{12 \ldots N}^{1^{\prime} 2^{\prime} \ldots N^{\prime}} f_{1^{\prime} 2^{\prime} \ldots N^{\prime}} .
$$

We will use the 1-particle operator $(A(2) \mid B(1))$ defined as follows:

$$
(A(2) \mid B(1))_{1^{\prime}}^{1}=A_{1^{\prime} 2^{\prime}}^{12} B_{2}^{2^{\prime}} .
$$

The operator $(A(2) \mid B(1))$ is linear in both components $A(2)$ and $B(1)$.

\section{The theory of the best multiconfigurational approximations}

\subsection{Hamiltonian of $\mathrm{N}$-fermion system}

Let us consider a quantum-mechanical system of $N$ identical fermions interacting via 2-body forces in the presence of an external field. The Hamiltonian of such a system has the following form:

$$
\begin{aligned}
H(N) & =H[1, \ldots, N] \\
= & \sum_{i=1}^{N} H[i] \otimes I[1, \ldots, i-1, i+1, \ldots, N] \\
+ & \sum_{1 \leq i<j \leq N} V[i, j] \otimes I[1, \ldots, i-1, i+1, \\
& \quad \ldots, j-1, j+1, \ldots, N] \\
= & \sum_{1 \leq i<j \leq N} H[i, j] \otimes I[1, \ldots, i-1, i+1, \\
& \quad \ldots, j-1, j+1, \ldots, N],
\end{aligned}
$$

where

$$
H[i, j]=\frac{H[i] \otimes I[j]+H[j] \otimes I[i]}{N-1}+V[i, j] .
$$

Because the Hamiltonian $H(N)$ describes a system of $N$ identical fermions we will consider its antisymmetrical projection onto $\mathcal{H}^{\wedge N}$, i.e.

$$
\begin{aligned}
& \hat{H}(N)=A(N) H(N) A(N)=A(N) H(N) \\
& \quad=H(N) A(N)=\left(\begin{array}{c}
N \\
2
\end{array}\right) \hat{H}(2) \wedge \hat{I}(N-2),
\end{aligned}
$$

where

$$
\hat{H}(2)=\frac{2}{N-1} F(1) \wedge I(1)+\hat{V}(2)
$$

and $\hat{V}(2)$ are antisymmetrical projections of $H(2)$ and $V(2)$ onto $\mathcal{H}^{\wedge 2}$.

Further, the Hamiltonian of a system of $N$ fermions $\hat{\mathcal{H}}(N)$ bounded from below and possessing discrete eigenvalues, at least in the lower part of the spectrum, is considered.

\subsection{The multiconfigurational analysis}

Let us consider a finite-dimensional subspace $\mathcal{M}$ in the Hilbert space $\mathcal{H} . \mathcal{M}^{\wedge N}=\mathcal{M} \wedge \ldots \wedge \mathcal{M} \subset \mathcal{H}^{\wedge N}$ is the linear hull of all antisymmetrized tensor products of $N$ 1-fermion functions from $\mathcal{M}$. We call $\mathcal{M}$ the basic manifold, $\mathcal{M}^{\wedge N}$ the multiconfigurational manifold generated by $\mathcal{M}$, and observe that from $\operatorname{dim} \mathcal{M}=m$ follows $\operatorname{dim} \mathcal{M}^{\wedge N}=\left(\begin{array}{c}m \\ N\end{array}\right)$.

Throughout the paper it is assumed that $\mathcal{M}^{\wedge N}$ lies in the domain $D(\hat{H}(N))$ of $\hat{H}(N)$. Suppose $E_{1}=$ 
$\inf \left\{E(f): f \in \mathcal{M}^{\wedge N}\right\}$ is the minimal value of the energy functional $E(f)=\langle f \mid \hat{H}(N) f\rangle /\langle f \mid f\rangle$ in $\mathcal{M}^{\wedge N}$ and denote by $\mathcal{K}_{1}$ the linear subspace of $\mathcal{M}^{\wedge N}$ on which $E(f)$ has the value $E_{1}$, so $\mathcal{K}_{1}=\left\{f \in M^{\wedge N}: E(f)=E_{1}\right\}$. The pair $\left(E_{1}, \mathcal{K}_{1}\right)$ will be called the first (or ground-) level of $\hat{H}(N)$ in $\mathcal{M}^{\wedge N}, E_{1}$ is the energy and $\mathcal{K}_{1}$ the subspace of this level. Similary we call the pair $\left(E_{2}, \mathcal{K}_{2}\right)$, with $E_{2}=\inf \left\{E(f): f \in \mathcal{M}^{\wedge N} \ominus \mathcal{K}_{1}\right\}$ and $\mathcal{K}_{2}=\{f \in$ $\left.\mathcal{M}^{\wedge N} \ominus \mathcal{K}_{1}: E(f)=E_{2}\right\}$, the second level of $\hat{H}(N)$ in $\mathcal{M}^{\wedge N}$. This process may be continued until

$$
\mathcal{M}^{\wedge N}=\oplus_{\alpha=1}^{a} \mathcal{K}_{\alpha} .
$$

The set $\left\{\left(E_{\alpha}, \mathcal{K}_{\alpha}\right)\right\}_{\alpha=1}^{a}$ represents the complete set of levels of $\hat{H}(N)$ in $\mathcal{M}^{\wedge N}$. Introduce the projectors $P: \mathcal{H} \rightarrow$ $\mathcal{M}, P_{\mathcal{M}}^{\wedge N}: \mathcal{H}^{\wedge N} \rightarrow \mathcal{M}^{\wedge N}, P_{\alpha}: \mathcal{M}^{\wedge N} \rightarrow \mathcal{K}_{\alpha}^{\mathcal{M}}$ Then Eq. (2.2.1) is equivalent to

$$
P_{\mathcal{M}}^{\wedge N}=\sum_{\alpha=1}^{a} P_{\alpha}
$$

and the operator $\sum_{\alpha=1}^{a} E_{\alpha} P_{\alpha}$ is identical to the operator $\hat{\mathcal{H}}(N)=P_{\mathcal{M}}^{\wedge N} \hat{H}(N) P_{\mathcal{M}}^{\wedge N}$ i.e.

$$
\hat{\mathcal{M}}(N)=\sum_{\alpha=1}^{a} E_{\alpha} P_{\alpha} .
$$

Following Kato [3], we will call the operator $\underset{\mathcal{M}}{\hat{H}}(N)$ the orthogonal projection of the Hamiltonian $\hat{H}(N)$ onto the state space $\mathcal{M}^{\wedge N}$.

Physically, the self-adjoint operator $\hat{\mathcal{H}}(N)$ represents the Hamiltonian $\hat{H}(N)$ with the states reduced to those in the manifold $\mathcal{M}^{\wedge N}$. In other words, the first level $\left(E_{1}, \mathcal{K}_{1}\right)$ of $\underset{\mathcal{M}}{\hat{H}}(N)$ may represent a useful approximation to the ground-level of $\hat{H}(N)$, and likely a number of lowest levels of $\hat{H}(N)$ may be approximated by a subset of levels of $\hat{\mathcal{H}}(N)$.

The above approximations are known as multiconfigurational approximations (or CI - configuration interaction approximations). We will also use the term $\mathcal{M}$ -approximations, both for brevity and for displaying their dependence on the basic manifold.

Two principal problems involved in the theory of multiconfigurational approximations are: (i) the problem of determining optimal basic manifolds $\mathcal{M}$ (so called minimizing basic manifolds), and (ii) the most effective method of determining the eigenvalues of $\underset{\mathcal{M}}{\hat{H}}(N)$.

\subsection{The minimizing basic manifold}

The minimizing basic manifold $\mathcal{M}$ is that which for fixed $m=\operatorname{dim} \mathcal{M}$ uniquely satisfies the condition

$$
\underset{\mathcal{M}}{E}[\hat{H}(N)]=\frac{\operatorname{Tr}\left(\hat{H}(N) P_{\mathcal{M}}^{\wedge N}\right)}{\operatorname{Tr} P_{\mathcal{M}}^{\wedge N}}=\frac{\operatorname{Tr} \underset{\mathcal{M}}{\hat{H}}(N)}{\left(\begin{array}{c}
m \\
N
\end{array}\right)}=\min .
$$

From Eqs. (2.3.1) and (2.2.3) we see that

$$
\underset{\mathcal{M}}{E}[\hat{H}(N)]=\frac{\sum_{\alpha=1}^{a} E_{\alpha}}{\left(\begin{array}{l}
m \\
N
\end{array}\right)} .
$$

It means that $\underset{\mathcal{M}}{E}[\hat{H}(N)]$ is the average energy of $\hat{H}(N)$ on $\mathcal{M}^{\wedge N}$. Therefore the minimizing basic manifold $\mathcal{M}$ minimizes the average energy of $\hat{H}(N)$ on $\mathcal{M}^{\wedge N}$.

The requirement that $\mathcal{M}$ be unique means that the admissible values of $m=\operatorname{dim} \mathcal{M}$ are not arbitrary in general, but run over some sequence of magic numbers characteristic of the interactions in the system (2.1.1). If $m=\operatorname{dim} \mathcal{M}$ is a magic number, it implies the invariance of the multiconfigurational manifold $\mathcal{M}^{\wedge N}$ under the symmetry group of $\hat{H}(N)$, hence $\hat{H}(N)$ is also invariant under the symmetry group of $\hat{H}(N)$.

\subsection{Generalized self-consistent field equations}

From Eqs. (2.3.1) and (2.1.3) it follows that:

$$
\begin{gathered}
\underset{\mathcal{M}}{E}[\hat{H}(N)]=\frac{\left(\begin{array}{c}
m \\
N
\end{array}\right)}{\left(\begin{array}{c}
m \\
2
\end{array}\right)} \operatorname{Tr}\left(\hat{H}(2) P_{\mathcal{M}}^{\wedge 2}\right) \\
=\frac{\left(\begin{array}{c}
m \\
N
\end{array}\right)}{\left(\begin{array}{c}
m \\
2
\end{array}\right)} \hat{H}_{1^{\prime} 2^{\prime}}^{12} P_{\mathcal{M}}^{1^{\prime}} P_{\mathcal{M}}^{2^{\prime}}, \\
\mathcal{M}
\end{gathered}
$$

where $P_{1}^{1^{\prime}}$ is the kernel of $\mathcal{M}$. Hence, the variation of the

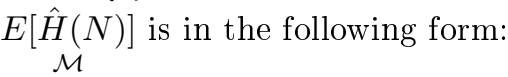

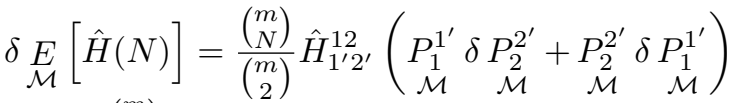

$$
\begin{aligned}
& =2 \frac{\left(\begin{array}{c}
m \\
N
\end{array}\right)}{\left(\begin{array}{c}
m \\
2
\end{array}\right)} \hat{H}_{1^{\prime} 2^{\prime}}^{12} P_{\mathcal{M}}^{2^{\prime}} \delta P_{1}^{1^{\prime}} .
\end{aligned}
$$

If $\{f\}_{i=1}^{m}$ is the orthonormal base in $\mathcal{M}$, then (i)

$$
\begin{aligned}
\underset{\mathcal{M}}{P_{1}^{1^{\prime}}}=\sum_{i=1}^{m} f_{(i)}^{1^{\prime}} \underset{(i)}{f_{1}} \text { and } \\
\left.\quad \delta P_{\mathcal{M}}^{1^{\prime}}=\sum_{i=1}^{m} \underset{(i)}{\left(f_{(i)}^{1^{\prime}}\right.} \delta \underset{(i)}{\delta} f_{(i)}+\underset{(i)}{f_{1} \delta f^{1^{\prime}}}\right) .
\end{aligned}
$$

Substituting this in Eq. (2.4.2) we find

$$
\frac{1}{2} \delta \underset{\mathcal{M}}{E}[\hat{H}(N)]=\sum_{i=1}^{m} K_{1^{\prime}}^{1} f_{(i)}^{1^{\prime}} \delta \underset{(i)}{f_{1}}+\sum_{i=1}^{m} K_{1^{\prime}}^{1} \underset{(i)}{f_{1}} \delta f_{(i)}^{1^{\prime}},
$$

where the 1-particle operator $K(1)$ has the form

$$
K(1)=\frac{\left(\begin{array}{c}
m \\
N
\end{array}\right)}{\left(\begin{array}{c}
m \\
2
\end{array}\right)}(\hat{H}(2) \mid \underset{\mathcal{M}}{P}) .
$$

In turn, the 1-particle operator $(\hat{H}(2) \mid \underset{\mathcal{M}}{P}) \quad(\mathrm{cf}$. Eq. (1.2.10)) is that whose kernel is defined as follows:

$$
(\hat{H}(2) \mid \underset{\mathcal{M}}{P})_{1^{\prime}}^{1}=\hat{H}_{1^{\prime} 2^{\prime}}^{12} P_{\mathcal{M}}^{2^{\prime}} .
$$

The minimum of $\underset{\mathcal{M}}{E}[\hat{H}(N)]$ under the conditions $f^{1} f_{1}=\delta_{i, j}$ is equivalent to (i) $(j)$ 


$$
\delta\left(\frac{1}{2} \underset{\mathcal{M}}{E}[\hat{H}(N)]-\sum_{i, j=1}^{m} \lambda_{j, i} \underset{(i)}{f_{1}} \underset{(j)}{f_{2}}\right)=0,
$$

where $\lambda_{j, i}$ are the Lagrange multipliers. Eqs. (2.4.4) and (2.4.7) we have

$$
\begin{gathered}
\sum_{i=1}^{m}\left[\left(K_{1^{\prime}}^{1} f_{(i)}^{1^{\prime}}-\sum_{j=1}^{m} \lambda_{j, i} \underset{(j)}{f_{(j)}^{1}}\right) \delta \underset{(i)}{f_{1}+\left(K_{1^{\prime}}^{1} f_{(i)}\right.}\right. \\
\left.\left.-\sum_{j=1}^{m} \lambda_{i, j} \underset{(j)}{f_{1^{\prime}}}\right) \delta f_{(i)}^{1^{\prime}}\right]=0 .
\end{gathered}
$$

Hence,

$$
K_{1^{\prime}}^{1} f_{(i)}^{1^{\prime}}=\sum_{j=1}^{m} \lambda_{j, i} f_{(j)}^{f^{1}}, \quad i=1,2, \ldots, m
$$

and

$$
K_{1^{\prime}}^{1} \underset{(i)}{f_{1}}=\sum_{j=1}^{m} \lambda_{i, j} \underset{(j)}{f_{1^{\prime}}}, \quad i=1,2, \ldots, m .
$$

Since $\hat{H}(2)$ is a self-adjoint operator, it follows that $K(1)$ is a self-adjoint operator and $\lambda_{j, i}=\overline{\lambda_{i, j}}$. Then Eqs. (2.4.9) are equivalent to Eqs. $\left(2.4 .9^{\prime}\right)$ and we may restrict ourselves to Eqs. (2.4.9). Equations (2.4.9) are necessary conditions for the minimizing basic manifold $\mathcal{M}$ and will be called the generalized self-consistent field equations [2]. In the case $m=N$ the generalized self-consistent field equations are equivalent to the HartreeFock equations $[4,5]$.

From Eqs. (2.4.9) we see that the minimizing basic manifold $\mathcal{M}$ is an invariant subspace of the operator $K(1)$. Hence, the operator $K(1)$ and the projector $\underset{\mathcal{M}}{P}$ commutate. i.e.

$$
[K(1), \underset{\mathcal{M}}{P}]=K(1) \underset{\mathcal{M}}{P}-\underset{\mathcal{M}}{P} K(1)=0 .
$$

Equation (2.4.10) is equivalent to Eqs. (2.4.9).

\subsection{The best multiconfigurational approximations} 2.5.1. The best 1-particle approximation

A set $\mathcal{S}(1)=\{X(1)=\bar{X}(1): \mathcal{M} \rightarrow \mathcal{M}\}$ of self-adjoint operators $X(1)$ in $\mathcal{M}$ is a real linear space, whose dimension is $m^{2}(m=\operatorname{dim} \mathcal{M})$. Hence a set $\mathcal{S}_{1}(N)=$ $N \mathcal{S}(1) \wedge P_{\mathcal{M}}^{\wedge(N-1)}=\left\{N X(1) \wedge P_{\mathcal{M}}^{\wedge(N-1)}: X(1) \in \mathcal{S}(1)\right\}$ is a real linear space of 1-particle observables in $\mathcal{M}^{\wedge N}$. By $\mathcal{S}(N)$ we denote $\left(\left(\begin{array}{c}m \\ N\end{array}\right)^{2}\right.$-dimensional) a real Hilbert space of all Hermitian operators in $\mathcal{M}^{\wedge N}$ with the scalar product

$$
\begin{gathered}
\langle X(N) \mid Y(N)\rangle=\operatorname{Tr}(X(N) Y(N)), \\
X(N), Y(N) \in \mathcal{S}_{\mathcal{M}}(N),
\end{gathered}
$$

then $\mathcal{S}_{1}(N)$ is a real linear subspace of $\mathcal{S}(N)$ and $\hat{\mathcal{M}}(N) \in \mathcal{S}_{\mathcal{M}}(N)$. As a result there exists one and only one element $\underset{1}{H}(N) \in \mathcal{S}_{1}(N)$ which minimizes the HilbertSchmidt norm

$$
\left.|\hat{H}(N)-\hat{H}(N)|=\left(\operatorname{Tr} \underset{\mathcal{M}}{(H}(N)-H_{1}(N)\right)^{2}\right)^{1 / 2}
$$

of $\underset{\mathcal{M}}{\hat{H}}(N)-\underset{1}{H}(N)$ induced by the scalar product (2.5.1) [6].
The operator $\hat{H}_{1}(N)$, so called the best 1-particle approximation of $\hat{\mathcal{H}}(N)$, has the form

$$
\hat{H}(N)=N L(1) \wedge P_{\mathcal{M}}^{\wedge(N-1)},
$$

where

$$
\begin{aligned}
L(1) & =\frac{(N-1)\left(\begin{array}{c}
m \\
2
\end{array}\right)}{(m-2)\left(\begin{array}{c}
m \\
N
\end{array}\right)} \\
\times & {\left[2 \underset{\mathcal{M}}{K}(1)-(m-1)^{-1}(\operatorname{Tr} \underset{\mathcal{M}}{K}(1)) \underset{\mathcal{M}}{P}\right] }
\end{aligned}
$$

is the so-called 1-particle component of $\hat{H}(N)$. Above $\underset{\mathcal{M}}{K}(1)=\underset{\mathcal{M}}{P} K(1) \underset{\mathcal{M}}{P}$, and $K(1)$ is given by (2.4.5).

The operator $\underset{1}{H}(N)$ has the following important properties:

(i) its average energy is equal to the average energy of the Hamiltonian $\hat{H}(N)$ on $\mathcal{M}^{\wedge N}$, i.e.

$$
\frac{\operatorname{Tr} \hat{H}(N)}{\operatorname{Tr} P^{\wedge N}}=\frac{\operatorname{Tr} \hat{H}(N) P^{\wedge N}}{\operatorname{Tr} P^{\wedge N}},
$$

(ii) if $\mathcal{M}$ is the minimizing base manifold, the operator $\underset{1}{H}(N)$ is invariant under the symmetry group of the Hamiltonian $\hat{H}(N)$.

The eigenstructure of $L(1)$ uniquely determines the eigenstructure of $\hat{H}_{1}(N)$. If $L(1)$ has eigenvalues $\mathcal{E}_{a}$ and eigenspaces $\mathcal{M}_{a}, a=1,2, \ldots, A$ the eigenvalues and eigenspaces of $\hat{H}(N)$ are given by the formulae

$$
E_{(1, \underline{N})}=\sum_{a=1}^{A} N_{a} \mathcal{E}_{a}, \quad \mathcal{M}^{\wedge} \underline{N}=\mathcal{M}_{1}^{\wedge N_{1}} \wedge \ldots \wedge \mathcal{M}_{A}^{\wedge N_{A}} .
$$

Above $N$ is the so called occupation-number vector which is defined as a sequence $\underline{N}=\left\{N_{a}\right\}_{a=1}^{A}$ of non-negative integers such that $0 \leq N_{a} \leq \operatorname{dim} \mathcal{M}_{a}, \sum_{a=1}^{A} N_{a}=N$. In turn $\mathcal{M}^{\wedge} \underline{N}$ is a natural configuration of the index $\underline{N}$ i.e. the linear hull of the Slater determinants of $N$ 1-particle functions among which there are exactly $N_{a}$ independent ones from $M_{a}$, for each $a$. Denoting by $P_{N}$ the projector onto $\mathcal{M}^{\wedge} \underline{N}$ we may now write the spectral decomposition of $\hat{H}(N)$ in the form

$$
\hat{H}(N)=\sum_{\underline{N}} E_{(1, \underline{N})} P_{\underline{N}} .
$$

\subsubsection{The best coarse-grained configurational approximation}

Now we modify the best 1-particle approximation getting a better approximation to $\hat{H}(N)$. To do this let us consider the real linear space $\mathcal{S}_{2}(N)$ of operators of the type

$$
X(N)=\sum_{\underline{N}} x_{\underline{N}} P_{\underline{N}},
$$

where $x_{N}$ are the real numbers. The $\mathcal{S}_{2}(N)$ is the subspace of the space $\mathcal{S}_{\mathcal{M}}(N)$ and therefore contains a unique operator $\hat{H}_{2}(N)$ which minimizes the Hilbert-Schmidt 
norm of $\underset{\mathcal{M}}{\hat{H}}(N)-\underset{2}{\hat{H}}(N)$, i.e. $|\underset{\mathcal{M}}{\hat{H}}(N)-\underset{2}{\hat{H}}(N)|=\min$. The operator $\underset{2}{H}(N)$ so called the best coarse-grained configurational approximation of $\hat{\mathcal{M}}(N)$ has the form $\sum_{\underline{N}} E_{(2, \underline{N})} P_{\underline{N}}$, where

$$
E_{(2, \underline{N})}=\frac{\operatorname{Tr}\left(\hat{H}(N) P_{\underline{N}}\right)}{\operatorname{Tr} P_{\underline{N}}}
$$

are eigenvalues of $\underset{2}{H}(N)$ and coincide with the average values of $\hat{H}(N)$ on eigenspaces $\mathcal{M}^{\wedge} \underline{N}$. The eigenvalues $E_{(2, \underline{N})}$ may also be written in terms of the configurational averages of the operator $\hat{H}(2)$ (see Eq. (2.1.4)), i.e.

$$
E_{(2, \underline{N})}=\sum_{a=1}^{A}\left(\begin{array}{c}
N_{a} \\
2
\end{array}\right) \mathcal{E}_{a, a}+\sum_{1 \leq a<b \leq A} \mathcal{E}_{a, b},
$$

where

$$
\mathcal{E}_{a, b}=\frac{\operatorname{Tr}\left(\hat{H}(2)\left(P_{a} \wedge P_{b}\right)\right)}{\operatorname{Tr}\left(P_{a} \wedge P_{b}\right)} .
$$

$P_{a}, a=1,2, \ldots, A$ are projectors onto eigenspaces $\mathcal{M}_{a}$, $a=1,2, \ldots, A$ of operator $L(1)$ given by Eq. (2.5.4).

The operator $\underset{2}{H}(N)$ has the following properties:

(i) its average energy on the natural configuration $\mathcal{M}^{\wedge} \underline{N}$ is equal to the average energy of the Hamiltonian $\hat{H}(N)$ on the same natural configuration, i.e.

$$
\frac{\operatorname{Tr}\left(\hat{H}(N) P_{\underline{N}}\right)}{\operatorname{Tr} P_{\underline{N}}}=\frac{\operatorname{Tr}\left(\hat{H}(N) P_{\underline{N}}\right)}{\operatorname{Tr} P_{\underline{N}}}
$$

(ii) if $\mathcal{M}$ is a minimizing base manifold, the operator $\hat{H}(N)$ is invariant under the symmetry group of $\hat{H}(N)$.

\section{Application of the best multiconfigurational approximations for atomic ions}

3.1. Hamiltonian of an atomic ion

Consider the non-relativistic Hamiltonian (in atomic units)

$$
\begin{aligned}
& H^{(N, Z)}=\sum_{i=1}^{N}-\left(\frac{1}{2} \Delta_{i}+\frac{Z}{r_{i}}\right)+\sum_{1 \leq i<j \leq N} \frac{1}{r_{i, j}} \\
& =\sum_{1 \leq i<j \leq N}\left\{-\frac{\left(\frac{1}{2} \Delta_{i}+\frac{Z}{r_{i}}\right)+\left(\frac{1}{2} \Delta_{j}+\frac{Z}{r_{j}}\right)}{N-1}+\frac{1}{r_{i, j}}\right\}
\end{aligned}
$$

of an atomic ion $(N, Z)$ with $N$ electrons and nuclear charge $Z$.

Rescaling the space coordinates, $\underline{r} \rightarrow \underline{r} / Z$ we obtain

$$
\begin{gathered}
H^{(N, Z)}=\frac{Z^{2}}{N-1} \sum_{1 \leq i<j \leq N} H_{\lambda}[i, j] \otimes I[1, \ldots, i-1, \\
i+1, \ldots, j-1, j+1, \ldots, N],
\end{gathered}
$$

where

$$
H_{\lambda}[i, j]=F[i]+F[j]+\lambda V[i, j] .
$$

Above $F[i]$ is the Hamiltonian of the hydrogen atom,
$V[i, j]$ is the Coulomb interaction potential between two electrons, i.e.

$$
\begin{aligned}
& F[i]=-\left(\frac{1}{2} \Delta_{i}+\frac{1}{r_{i}}\right), \\
& V[i, j]=\frac{1}{r_{i, j}}
\end{aligned}
$$

and $\lambda=\frac{N-1}{Z}$ is the reciprocal of the reduced nuclear charge $\xi=\frac{Z}{N-1}$ [2]. From Eq. (3.1.2) the antisymmetrical projection $\hat{H}^{(N, Z)}$ of $H^{(N, Z)}$ is in the following form:

$$
\hat{H}^{(N, Z)}=\frac{Z^{2}}{N-1}\left(\begin{array}{c}
N \\
2
\end{array}\right) \hat{H}_{\lambda}(2) \wedge \hat{I}(N-2),
$$

where

$$
\hat{H}_{\lambda}(2)=2 F(1) \wedge I(1)+\lambda \hat{V}(2)
$$

is the antisymmetrical projection of $H_{\lambda}[i, j]$ onto $\mathcal{H}^{\wedge 2}$.

3.2. The minimizing basic manifold of $\hat{H}^{(N, Z)}$ and its canonical decomposition

The minimizing basic manifold of the Hamiltonian $\hat{H}^{(N, Z)}$ fulfills the variational condition (cf. Eqs. (2.3.1), (3.1.6), (2.4.1)):

$$
\begin{gathered}
\underset{\mathcal{M}}{E}\left[\hat{H}^{(N, Z)}\right]=\frac{\operatorname{Tr}\left(\hat{H}^{(N, Z)} P_{\mathcal{M}}^{\wedge N}\right)}{\operatorname{Tr} P_{\mathcal{M}}^{\wedge N}} \\
=\frac{Z^{2}}{N-1} \frac{\left(\begin{array}{c}
m \\
N
\end{array}\right)}{\left(\begin{array}{c}
m \\
2
\end{array}\right)} \operatorname{Tr}\left(\hat{H}_{\lambda}(2) P_{\mathcal{M}}^{\wedge 2}\right)=\min .
\end{gathered}
$$

From Eq. (3.2.1) follows that the ions $(N, Z)$ having the same reduced nuclear charge $\xi=\lambda^{-1}$, have the same minimizing basic manifolds $\mathcal{M}_{\lambda}$.

The necessary condition for the minimizing basic manifolds $\mathcal{M}_{\lambda}$ of $\hat{H}^{(N, Z)}$ have the form (cf. Eqs. (3.2.1), (2.4.10), (2.4.5), (2.4.6)):

$$
\left[\left(\hat{H}_{\lambda}(2) \mid P_{\lambda}\right), P_{\lambda}\right]=0
$$

where $P_{\lambda}$ is the projector onto $\mathcal{M}_{\lambda}$. Operators $\left(\hat{H}_{\lambda}(2) \mid P_{\lambda}\right)$ and $P_{\lambda}$ commutate.

Equation (3.2.2) will be solved by means of canonical transformations $[3,7]$ with the perturbation parameter $\lambda$, which for atomic ions satisfies the inequality $0 \leq \lambda<1$. In the case of vanishing interactions, i.e. $\lambda=0$ the Hamiltonian of the system takes the form $\hat{H}^{(N, Z)}=Z^{2} N F(1) \wedge \hat{I}(N-1)$ which denotes the Hamiltonian of $N$ non-interacting electrons moving in the field of the atomic nucleus with the charge $Z$. For $\lambda=0$ the minimizing basic manifolds may be calculated immediately from the variational principle (3.2.1), which may be rewritten as follows:

$$
\operatorname{Tr} F(1) P_{0}=\min .
$$

From Eq. (3.2.3) we can see that the projectors $P_{0}^{(k)}$ on the minimizing basic manifolds $\mathcal{M}_{0}^{(k)}$ are in the form

$$
P_{0}^{(k)}=\sum_{n=1}^{k} R_{0}^{(n)}, \quad k=1,2,3, \ldots
$$

where $R_{0}^{(n)}$ is the projector on the hydrogen atom eigenspace corresponding to the eigenvalue $e_{n}=-\frac{1}{2 n^{2}}$. 
In the case $\lambda \neq 0$ we seek the minimizing basic manifold $\mathcal{M}_{\lambda}^{(k)}$ in the following form:

$$
\mathcal{M}_{\lambda}^{(k)}=P_{\lambda}^{(k)} \mathcal{H}
$$

where the projector $P_{\lambda}^{(k)}$ on $\mathcal{M}_{\lambda}^{(k)}$ is a canonical transformation of $P_{0}^{(k)}$, i.e.

$$
P_{\lambda}^{(k)}=U_{\lambda} P_{0}^{(k)} U_{\lambda}^{+}
$$

and $\mathcal{H}$ is the Hilbert space of square integrable 1-electrons functions including spin. For the canonical transformation $U_{\lambda}$ we postulate the following form:

$$
U_{\lambda}=\exp \left(\mathrm{i}\left(\lambda D_{1}+\lambda^{2} D_{2}+\lambda^{3} D_{3}+\ldots\right)\right),
$$

where $D_{j}, j=1,2, \ldots$ are self-adjoints operators.

There is a class of operators $U_{\lambda}$ in the form (3.2.7) which by (3.2.6) satisfy Eq. (3.2.2). We denote the projector on the common eigenspace of the Hamiltonian of the hydrogen atom $F(1)$ and of the operator of the square of the angular momentum corresponding to the eigenvalues $e_{n}=\frac{-1}{2 n^{2}}$ and $l(l+1)$ by $R_{0}^{(n, l)}$. The requirement that

$$
R_{\lambda}^{(n, l)}=U_{\lambda} R_{0}^{(n, l)} U_{\lambda}^{+}, \quad 0 \leq l<n \leq k
$$

are projectors on invariants subspaces of the operator $\left(\hat{H}_{\lambda}(2) \mid P_{\lambda}^{(k)}\right)$, i.e.

$$
\left[\left(\hat{H}_{\lambda}(2) \mid P_{\lambda}^{(k)}\right), R_{\lambda}^{(n, l)}\right]=0, \quad 0 \leq l<n \leq k
$$

restrict considerations to the canonical transformations invariant under the three-dimensional rotation group of the system.

The transition from Eq. (3.2.2) to Eqs. (3.2.9) is connected with the transition from an arbitrary unitary transformation $U_{\lambda}: \mathcal{M}_{0}^{(k)}=P_{0}^{(k)} \mathcal{H} \rightarrow \mathcal{M}_{\lambda}^{(k)}=P_{\lambda}^{(k)} \mathcal{H}$ to the unitary transformation $U_{\lambda}: \mathcal{M}_{0}^{(n, l)}=R_{0}^{(n, l)} \mathcal{H} \rightarrow$ $\mathcal{M}_{\lambda}^{(n, l)}=R_{\lambda}^{(n, l)} \mathcal{H}$ preserving the decomposition of the minimizing basic manifold $\mathcal{M}_{\lambda}^{(k)}$ on the subspaces $\mathcal{M}_{\lambda}^{(n, l)}$, $0 \leq l<n \leq k$ which are non-reduced under the three-dimensional rotation group of the system. Indeed, from the relations

$$
R_{0}^{(n)}=\sum_{l=0}^{n-1} R_{0}^{(n, l)},
$$

and Eqs. (3.2.4), (3.2.6), (3.2.8) it follows that:

$$
P_{\lambda}^{(k)}=\sum_{n=1}^{k} \sum_{l=0}^{n-1} R_{\lambda}^{(n, l)}
$$

or equivalently

$$
\mathcal{M}_{\lambda}^{(k)}=\sum_{n=1}^{k} \sum_{l=0}^{n-1} \mathcal{M}_{\lambda}^{(n, l)} .
$$

The subspaces $\mathcal{M}_{\lambda}^{(n, l)}$ are the eigenspaces of the 1-particle component of the best 1-particle approximation of $\hat{H}^{(N, Z)}$. Indeed, the best 1-particle approximation of $\hat{H}^{(N, Z)}$ has the following form:

$$
\hat{H}_{\lambda}^{(N, Z)}=N L_{\lambda}(1) \wedge\left(P_{\lambda}^{(k)}\right)^{\wedge(N-1)},
$$

where the 1-particle component $L_{\lambda}(1)$ has the form

$$
\begin{aligned}
& L_{\lambda}(1)=\frac{Z^{2}}{m_{k}-2}\left[2 P_{\lambda}^{(k)}\left(\hat{H}_{\lambda}(2) \mid P_{\lambda}^{(k)}\right) P_{\lambda}^{(k)}\right. \\
& \left.-\left(m_{k}-1\right)^{-1} \operatorname{Tr}\left(P_{\lambda}^{(k)}\left(\hat{H}_{\lambda}(2) \mid P_{\lambda}^{(k)}\right)\right) P_{\lambda}^{(k)}\right],
\end{aligned}
$$

where the magic number $m_{k}=\operatorname{dim} \mathcal{M}_{\lambda}^{(k)}=\operatorname{Tr} P_{\lambda}^{(k)}=$ $\frac{1}{3} k(k+1)(2 k+1)$. From Eqs. (3.2.13), (3.2.11) and (3.2.9) we see that $\mathcal{M}_{\lambda}^{(n, l)}$ are eigenspaces of $L_{\lambda}(1)$. In turn the eigenvalues of $L_{\lambda}(1)$ are in the following form:

$$
\begin{aligned}
e_{\lambda}^{(n, l)} & =\frac{\operatorname{Tr}\left(L_{\lambda}(1) R_{\lambda}^{(n, l)}\right)}{\operatorname{Tr} R_{\lambda}^{(n, l)}} \\
& =\frac{Z^{2}}{m_{k}-2}\left[\operatorname{Tr}\left(\left(\hat{H}_{\lambda}(2) \mid P_{\lambda}^{(k)}\right) R_{\lambda}^{(n, l)}\right)(2 l+1)^{-1}\right. \\
& -\left(m_{k}-1\right)^{-1} \operatorname{Tr}\left(P_{\lambda}^{(k)}\left(\left(\hat{H}_{\lambda}(2) \mid P_{\lambda}^{(k)}\right)\right)\right] .
\end{aligned}
$$

Because $\mathcal{M}_{\lambda}^{(n, l)}, 0 \leq l<n \leq k$ are the eigenspaces of $L_{\lambda}(1)$, the decomposition $\left(3.2 .11^{\prime}\right)$ will be called the canonical decomposition of the minimizing basic manifold $\mathcal{M}_{\lambda}^{(k)}$.

\subsection{Determining the canonical transformation $U_{\lambda}$}

Equations (3.2.9) may be rewritten in the following form:

$$
\left[U_{\lambda}^{+}\left(\hat{H}_{\lambda}(2) \mid P_{\lambda}^{(k)}\right) U_{\lambda}, R_{0}^{(n, l)}\right]=0, \quad 0 \leq l<n \leq k
$$

or equivalently

$$
\begin{gathered}
R_{0}^{\left(n^{\prime}, l^{\prime}\right)} U_{\lambda}^{+}\left(\hat{H}_{\lambda}(2) \mid P_{\lambda}^{(k)}\right) U_{\lambda} R_{0}^{\left(n^{\prime \prime}, l^{\prime \prime}\right)}=0, \\
\left(n^{\prime}, l^{\prime}\right) \neq\left(n^{\prime \prime}, l^{\prime \prime}\right), \quad \min \left(n^{\prime}, n^{\prime \prime}\right) \leq k .
\end{gathered}
$$

By substituting the canonical transformation $U_{\lambda}=$ $\exp \left(\mathrm{i}\left(\lambda D_{1}+\lambda^{2} D_{2}+\lambda^{3} D_{3}+\ldots\right)\right)$ into $U_{\lambda}^{+}\left(\hat{H}_{\lambda}(2) \mid P_{\lambda}^{(k)}\right) U_{\lambda}$ and expanding it in powers of $\lambda$ one obtains

$$
\begin{aligned}
& U_{\lambda}^{+}\left(\hat{H}_{\lambda}(2) \mid P_{\lambda}^{(k)}\right) U_{\lambda}=\frac{1}{2}\left(m_{k} F(1)-F(1) P_{0}^{(k)}\right. \\
& \left.\quad-P_{0}^{(k)} F(1)+\left(\operatorname{Tr} F(1) P_{0}^{(k)}\right) I(1)\right) \\
& \quad+\sum_{j=1}^{\infty} \lambda^{j}\left\{\frac { 1 } { 2 } \left[m_{k} F_{j}(1)-F_{j}(1) P_{0}^{(k)}-P_{0}^{(k)} F_{j}(1)\right.\right. \\
& \left.\left.\quad+\left(\operatorname{Tr} F_{j}(1) P_{0}^{(k)}\right) I(1)\right]+\left(\hat{V}_{j-1}(2) \mid P_{0}^{(k)}\right)\right\},
\end{aligned}
$$

where

$$
\begin{gathered}
F_{j}(1)=\sum_{p=1}^{j} \frac{(-i)^{p}}{p !} \sum_{j_{1}+\ldots+j_{p}=j}\left[D_{j_{1}},\left[D_{j 2}, \ldots\right.\right. \\
\left.\left.\ldots\left[D_{j_{p}}, F(1)\right] \ldots\right]\right], \quad j=1,2,3, \ldots
\end{gathered}
$$

and

$$
\begin{aligned}
& \hat{V}_{0}(2)=\hat{V}(2), \\
& \hat{V}_{j}(2)=\sum_{p=1}^{j} \frac{(-i)^{p}}{p !} \sum_{j_{1}+\ldots+j_{p}=j}\left[G_{j_{1}},\left[G_{j 2}, \ldots\right.\right. \\
& \left.\left.\ldots\left[G_{j_{p}}, \hat{V}(2)\right] \ldots\right]\right], \quad j=1,2,3, \ldots
\end{aligned}
$$
where

$$
G_{j}=2 D_{j} \wedge I(1), \quad j_{1} \geq 1, \ldots, j_{p} \geq 1 .
$$

From Eqs. (3.3.1') and (3.3.2) it immediately follows that 
for $\left(n^{\prime}, l^{\prime}\right) \neq\left(n^{\prime \prime}, l^{\prime \prime}\right), \min \left(n^{\prime}, n^{\prime \prime}\right)<k$ :

$$
\begin{aligned}
& R_{0}^{\left(n^{\prime}, l^{\prime}\right)}\left\{m_{k} F(1)-F(1) P_{0}^{(k)}-P_{0}^{(k)} F(1)\right. \\
& \left.\quad+\left[\operatorname{Tr} F(1) P^{(k)}\right] I(1)\right\} R_{0}^{\left(n^{\prime \prime}, l^{\prime \prime}\right)}=0
\end{aligned}
$$

and

$$
\begin{aligned}
& R_{0}^{\left(n^{\prime}, l^{\prime}\right)}\left\{m_{k} F_{j}(1)-F_{j}(1) P_{0}^{(k)}-P_{0}^{(k)} F_{j}(1)\right. \\
& \left.+\left[\operatorname{Tr} F_{j}(1) P_{0}^{(k)}\right] I(1)+2\left(\hat{V}_{j-1}(2) \mid P_{0}^{(k)}\right)\right\} R_{0}^{\left(n^{\prime \prime}, l^{\prime \prime}\right)}=0 .
\end{aligned}
$$

Equation (3.3.4) is an identity, because the operators $P_{0}^{(k)}, R_{0}^{(n, l)}$ commutate with the Hamiltonian $F(1)$.

Substituting Eq. (3.3.3) into Eq. (3.3.4') and using the relation

$$
F(1) R_{0}^{(n, l)}=R_{0}^{(n, l)} F(1)=e_{n} R_{0}^{(n, l)}
$$

we obtain

$$
R_{0}^{\left(n^{\prime}, l^{\prime}\right)} D_{j} R_{0}^{\left(n^{\prime \prime}, l^{\prime \prime}\right)}=\frac{R_{0}^{\left(n^{\prime}, l^{\prime}\right)} T_{j}^{\left(n^{\prime}, n^{\prime \prime}\right)} R_{0}^{\left(n^{\prime \prime}, l^{\prime \prime}\right)}}{e_{n^{\prime}}-e_{n^{\prime \prime}}},
$$

where

$$
\begin{aligned}
& T_{1}^{\left(n^{\prime}, n^{\prime \prime}\right)}=\frac{2 \mathrm{i}\left(\hat{V}(2) \mid P_{0}^{(k)}\right)}{m_{k}-\Theta_{k, n^{\prime}}-\Theta_{k, n^{\prime \prime}}}, \\
& T_{j}^{\left(n^{\prime}, n^{\prime \prime}\right)}=\sum_{p=2}^{j} \frac{(-i)^{p-1}}{p !} \sum_{j_{1}+\ldots+j_{p}=j}\left[D_{j_{1}},\left[D_{j 2}, \ldots\right.\right. \\
& \left.\left.\quad \ldots\left[D_{j_{p}}, F(1)\right] \ldots\right]\right]+\frac{2 \mathrm{i}\left(\hat{V}_{j-1}(2) P_{0}^{(k)}\right)}{m_{k}-\Theta_{k, n^{\prime}}-\Theta_{k, n^{\prime \prime}}}, \\
& \quad j=2,3,4, \ldots
\end{aligned}
$$

and

$$
j_{1} \geq 1, \ldots, j_{p} \geq 1, \quad \Theta_{k, n}= \begin{cases}0, & k<n, \\ 1, & k \geq n .\end{cases}
$$

For $n^{\prime} \neq n^{\prime \prime}$, we sum up both sides of Eq. (3.3.6) over $l^{\prime}$ and $l^{\prime \prime}\left(0 \leq l^{\prime}<n^{\prime}, 0 \leq l^{\prime \prime}<n^{\prime \prime}\right)$ and obtain

$$
R_{0}^{\left(n^{\prime}\right)} D_{j} R_{0}^{\left(n^{\prime \prime}\right)}=\frac{R_{0}^{\left(n^{\prime}\right)} T_{j}^{\left(n^{\prime}, n^{\prime \prime}\right)} R_{0}^{\left(n^{\prime \prime}\right)}}{e_{n^{\prime}}-e_{n^{\prime \prime}}},
$$$$
\min \left(n^{\prime}, n^{\prime \prime}\right) \leq k .
$$

Equations (3.3.7) determine uniquely the non-diagonal blocks $R_{0}^{\left(n^{\prime}\right)} D_{j} R_{0}^{\left(n^{\prime \prime}\right)}$ of the operators $D_{j}, j=1,2, \ldots$, which are in turn determined by the blocks $R_{0}^{\left(n^{\prime}\right)} D_{j} R_{0}^{\left(n^{\prime \prime}\right)}$ of operators $D_{t}, t=1,2, \ldots, j-1$.

The minimal operator $D_{j}$ in the sense of the HilbertSchmidt norm which fulfils Eqs. (3.3.7) has diagonal blocks equal to zero, i.e. $R_{0}^{(n)} D_{j} R_{0}^{(n)}=0$. Hence

$$
D_{j}=\sum_{\substack{n^{\prime} \neq n^{\prime \prime} \\ \min \left(n^{\prime}, n^{\prime \prime}\right) \leq k}} \frac{R_{0}^{\left(n^{\prime}\right)} T_{j}^{\left(n^{\prime}, n^{\prime \prime}\right)} R_{0}^{\left(n^{\prime \prime}\right)}}{e_{n^{\prime}}-e_{n^{\prime \prime}}} .
$$

Operators $D_{j}$ have the property:

(i) they are invariant under the three-dimensional rotation group,

(ii) their Hilbert-Schmidt norms are finite.

\subsection{Approximate energy levels of the atomic ions}

We will calculate the approximate energy levels of the Hamiltonians $\hat{H}^{(N, Z)}$ of atomic ions $(N, Z)$. In the first approximation the energy levels of $\hat{H}^{(N, Z)}$ are treated as eigenvalue of the best 1-particle approximation of $\hat{H}^{(N, Z)}$ and in the second approximation the energy levels of $\hat{H}^{(N, Z)}$ are treated as the eigenvalues of the best coarse-grained configurational approximation of $\hat{H}^{(N, Z)}$.

\subsubsection{Eigenvalues of the best 1-particle approximation}

According to Eqs. (3.2.14), (3.2.11) and (3.2.8) the eigenvalues $e_{\lambda}^{(n, l)}$ of the 1-particle component $L_{\lambda}(1)$ are in the following form:

$$
\begin{aligned}
& e_{\lambda}^{(n, l)}=\frac{Z^{2}}{m_{k}-2}\left\{( 2 l + 1 ) \sum _ { n _ { 1 } = 1 } ^ { k } \sum _ { l _ { 1 } = 0 } ^ { n _ { 1 } - 1 } \operatorname { T r } \left(U _ { \lambda } ^ { + } \left(\hat{H}_{\lambda}(2)\right.\right.\right. \\
& \left.\left.\left.\mid R_{\lambda}^{\left(n_{1}, l_{1}\right)}\right) U_{\lambda} R_{0}^{(n, l)}\right)\right)-\left(m_{k}-2\right)^{-1} \\
& \quad \times \sum_{n_{1}=1}^{k} \sum_{l_{1}=0}^{n_{1}-1} \sum_{n_{2}=1}^{k} \sum_{l_{2}=0}^{n_{2}-1} \operatorname{Tr}\left(U_{\lambda}^{+}\left(\hat{H}_{\lambda}(2) \mid R_{\lambda}^{\left(n_{1}, l_{1}\right)}\right)\right. \\
& \left.\left.\left.\quad \times U_{\lambda} R_{0}^{\left(n_{2}, l_{2}\right)}\right)\right)\right\} .
\end{aligned}
$$

Similarly as Eq. (3.3.2) we obtain

$$
\begin{aligned}
& U_{\lambda}^{+}\left(\hat{H}_{\lambda}(2) \mid R_{\lambda}^{(n, l)}\right) U_{\lambda}=\frac{1}{2}\{2(2 l+1) F(1) \\
& \left.\quad-2 F(1) R_{0}^{(n, l)}+\left[\operatorname{Tr}\left(F(1) R_{0}^{(n, l)}\right)\right] I(1)\right\} \\
& \quad+\sum_{j=1}^{\infty} \lambda^{j}\left\{\frac { 1 } { 2 } \left[2(2 l+1) F_{j}(1)-F_{j}(1) R_{0}^{(n, l)}\right.\right. \\
& \left.\quad-R_{0}^{(n, l)} F_{j}(1)+\left(\operatorname{Tr}\left(F_{j}(1) R_{0}^{(n, l)}\right)\right) I(1)\right] \\
& \left.\quad+\left(\hat{V}_{j-1}(2) \mid R_{0}^{(n, l)}\right)\right\} .
\end{aligned}
$$

Then the expansion of $\operatorname{Tr}\left(U_{\lambda}^{+}\left(\hat{H}_{\lambda}(2) \mid R_{\lambda}^{\left(n_{1} l_{1}\right)}\right) U_{\lambda} R_{0}^{\left(n_{2}, l_{2}\right)}\right)$ in powers $\lambda$ is in the following form:

$$
\begin{aligned}
\operatorname{Tr} & \left(U_{\lambda}^{+}\left(\hat{H}_{\lambda}(2) \mid R_{0}^{\left(n_{1}, l_{1}\right)}\right) U_{\lambda} R_{0}^{\left(n_{2}, l_{2}\right)}\right) \\
& =e_{0}\left(n_{1}, l_{1}, n_{2}, l_{2}\right)+\lambda e_{1}\left(n_{1}, l_{1}, n_{2}, l_{2}\right) \\
& +\lambda^{2} e_{2}\left(n_{1}, l_{1}, n_{2}, l_{2}\right)+\ldots,
\end{aligned}
$$

where

$$
\begin{aligned}
& e_{0}\left(n_{1}, l_{1}, n_{2}, l_{2}\right)=\left(2 l_{1}+1\right) \operatorname{Tr}\left(F(1) R_{0}^{\left(n_{2}, l_{2}\right)}\right) \\
& \quad+\left(2 l_{2}+1\right) \operatorname{Tr} F(1) R_{0}^{\left(n_{1}, l_{1}\right)} \\
& \quad-\operatorname{Tr}\left(F(1) R_{0}^{\left(n_{1}, l_{1}\right)}\right) \delta_{n_{1}, n_{2}} \delta_{l_{1}, l_{2}}, \\
& \quad e_{j}\left(n_{1}, l_{1}, n_{2}, l_{2}\right)=\left(2 l_{1}+1\right) \operatorname{Tr}\left(F_{j}(1) R_{0}^{\left(n_{2}, l_{2}\right)}\right) \\
& \quad-\operatorname{Tr}\left(F_{j}(1) R_{0}^{\left(n_{1}, l_{1}\right)}\right) \delta_{n_{1}, n_{2}} \delta_{l_{1}, l_{2}} \\
& \quad+\left(2 l_{2}+1\right) \operatorname{Tr}\left(F_{j}(1) R_{0}^{\left(n_{1}, l_{1}\right)}\right) \\
& \quad+\operatorname{Tr}\left(\left(\hat{V}_{j-1}(2) \mid R_{0}^{\left(n_{1}, l_{1}\right)}\right) R_{0}^{\left(n_{2}, l_{2}\right)}\right), \quad j \geq 1 .
\end{aligned}
$$

Above $\operatorname{Tr}\left(F(1) R_{0}^{(n, l)}\right)=2(2 l+1) e_{n}$.

From Eqs. (3.4.1) and (3.4.3) one obtains

$$
e_{\lambda}^{(n, l)}=e_{0}(n, l)+\lambda e_{1}(n, l)+\lambda^{2} e_{2}(n, l)+\ldots,
$$


where

$$
\begin{aligned}
& e_{0}(n, l)=-\frac{1}{2} \frac{Z^{2}}{n^{2}}, \\
& e_{i}(n, l)=\frac{Z^{2}}{m_{k}-2}\left\{(2 l+1)^{-1} \sum_{n_{1}=1}^{k} \sum_{l_{1}=0}^{n_{1}-1} e_{i}\left(n_{1}, l_{1}, n, l\right)\right. \\
& \left.\quad-\left(m_{k}-1\right)^{-1} \sum_{n_{1}=1}^{k} \sum_{l_{1}=0}^{n_{1}-1} \sum_{n_{2}=1}^{k} \sum_{l_{2}=0}^{n_{2}-1} e_{i}\left(n_{1}, l_{1}, n_{2}, l_{2}\right)\right\}, \\
& i=1,2,3, \ldots
\end{aligned}
$$

Finally, the eigenvalues of the best 1-particle approximation (cf. Eqs. (2.5.6), (3.4.5)) are in the following form:

$$
E_{(1, \underline{N})}=E_{(1, \underline{N})}^{(0)}+\lambda E_{(1, \underline{N})}^{(1)}+\lambda^{2} E_{(1, \underline{N})}^{(2)}+\ldots,
$$
where

$$
E_{(1, \underline{N})}^{(i)}=\sum_{n=1}^{k} \sum_{l=0}^{n-1} N_{(n, l)} e_{i}(n, l), \quad i=1,2,3 \ldots
$$

and the occupation number vector

$$
\begin{gathered}
\underline{N}=\left\{N_{(n, l)}: 0 \leq l<n \leq k, 0 \leq N_{(n, l)}<2(2 l+1),\right. \\
\left.\sum_{n=1}^{k} \sum_{l=0}^{n-1} N_{(n, l)}=N\right\} .
\end{gathered}
$$

\subsubsection{Eigenvalues of the best coarse-grained} approximation

The final formula for the approximation energies of atomic ions in the case of the best coarse-grained configurational approximation (cf. Eq. (2.5.10)) is in the following form:

$$
E_{(2, \underline{N})}=E_{(2, \underline{N})}^{(0)}+\lambda E_{(2, \underline{N})}^{(1)}+\lambda^{2} E_{(2, \underline{N})}^{(2)}+\ldots,
$$
where

$$
\begin{aligned}
& E_{(2, \underline{N})}^{(i)}=\frac{Z^{2}}{N-1}\left[\sum_{n=1}^{k} \sum_{l=0}^{n-1} \frac{\left(\begin{array}{c}
N_{(n, l)} \\
2
\end{array}\right)}{(2 l+1)(4 l+1)} e_{i}(n, l, n, l)\right. \\
& \quad+\sum_{n_{1}=1}^{k} \sum_{l_{1}=0}^{n_{1}-1} \sum_{\substack{n_{2}=1 \\
n_{2} \neq n_{1}}}^{k} \sum_{\substack{l_{2}=0 \\
l_{2} \neq l_{1}}}^{n_{2}-1} \frac{N_{\left(n_{1}, l_{1}\right)} N_{\left(n_{2}, l_{2}\right)}}{4\left(2 l_{1}+1\right)\left(2 l_{2}+1\right)} \\
& \left.\quad \times e_{i}\left(n_{1}, l_{1}, n_{2}, l_{2}\right)\right] .
\end{aligned}
$$

\section{Concluding remarks}

In this paper we have calculated the formulae for approximate eigenvalues and eigenspaces of lowest energy states of the atomic ions using theory of the best multiconfigurational approximations.

Both the basic tasks of this method, i.e. problem of determining the minimizing basic manifold and of determining the approximate energy levels and eigenspaces are connected with one another, by the canonical transformation which transforms the minimizing base manifold and eigenspaces of the unperturbed system (i.e. with the interactions between electrons neglected) into the minimizing base manifold and eigenspaces of the perturbed system.
It was shown that the Hamiltonian $\hat{H}^{(N, Z)}$ is proportional to the operator $\hat{H}_{\lambda}(2)$ (cf. Eq. (3.1.6)). Hence, all atomic ions having the same parameter $\lambda$ have the same minimizing basic manifolds and eigenspaces (in unit $Z^{-1}$ ).

The final formulae for energy levels in 1-particle and coarse-grained approximation are in the form of a power series of $\lambda$. The coefficients of the both power series $E_{(1, \underline{N})}^{(i)}$ and $E_{(2, \underline{N})}^{(i)}$ are simply expressed by the coefficients $e_{i}\left(n_{1}, l_{1}, n_{2}, l_{2}\right)$ (cf. Eq. (3.4.4)).

In separated work author with coworkers will present numerical calculations of energy levels of atomic ions (cf. Eqs. (3.4.8), (3.4.11)) and its dependence on the dimension of the minimizing basic manifold and number of components in power series (3.4.8), (3.4.11). Obtained numerical data will be compared with experimental data.

\section{References}

[1] S. Pruski, "Generalized one-particle analysis of systems of $n$ fermions", Lecture given at 5th Symp. on Mathematical Physics, Toruń, December 1973.

[2] S. Pruski, Physica 37, 246 (1967).

[3] T. Kato, Perturbation Theory for Linear Operators, Springer, Berlin 1966.

[4] D.R. Hartree, Proc. Camb. Philos. Soc. 24, 89 (1928).

[5] V.A. Fock, Z. Phys. 61, 126 (1930).

[6] A. Akhieser, I.M. Glazman, Theory of linear operators in Hilbert space, Frederick Ungar Publishing Company, New York 1961.

[7] D.J. Klein, J. Chem. Phys. 61, 786 (1974). 